\title{
INTERFACE THERMAL CONDUCTANCE FOR MICROACTUATED DEVICES
}

\author{
Woo-Bin Song and Joseph J. Talghader \\ Department of Electrical and Computer Engineering, University of Minnesota \\ Minneapolis, MN 55455
}

\begin{abstract}
In this paper, the thermal contact conductance (TCC) of actuated (snapped-down) interfaces is characterized, perhaps for the first time. The TCC of a typical polysilicon/nitride interface brought together by electrostatic actuation is about $6.0 \mathrm{~W} /\left(\mathrm{K}-\mathrm{cm}^{2}\right)$ in air and $9.5 \mathrm{~W} /\left(\mathrm{K}-\mathrm{cm}^{2}\right)$ under vacuum. For every device tested, the TCC is higher in vacuum than in air. This is in stark contrast to the behavior of bulk interfaces and may be the result of decreased solid-solid contact area in air caused by the pressure of the interstitial fluid. It is also found that the TCC increases with electrostatic pressure, as expected from bulk theory, but appears to be largely unaffected by monolayer surface treatments.
\end{abstract}

\section{INTRODUCTION}

The thermal contact conductance is the most critical parameter in the study of heat transfer across interfaces. While the TCC has been exhaustively studied for interfaces in microelectronics packaging[1], the TCC of MEMS interfaces has not been explored, perhaps because standard measurement techniques are difficult to apply in surface micromachining. Despite this, the design of many micromachined devices such as AFM thermal probes[2] and uncooled IR detectors[3] could be significantly improved by understanding their TCCs. In addition, recent work has shown the possibility of creating micromechanical devices with electronically tunable thermal conductance [4], where the tuning range would be greatly extended with the high TCCs measured here.

Theoretical models for the TCC indicate that there are two primary paths for heat conduction between two interfaces at room temperature[5]: one is solid-solid conduction through the contact points, and the other is conduction through an interstitial material, if there is one. Measurements of macroscopic interfaces have shown that TCCs measured in vacuum are significantly lower than those measured with an interstitial gas, usually air[6]. This behavior is typically attributed to the additional conduction paths offered by the air. To date, the modeling and experimentation for the TCC have been developed for interfaces that have surface roughness on the order of a micron or so. Little or no data appears to exist on the TCC of interfaces with roughness multiple orders of magnitude less than this value. However, the mechanical interface between a micromachined device and its underlying substrate, as shown in Fig. 1, may have surface roughness on the order of a few nanometers or less. In this paper, it is shown that the TCC of micromechanical interfaces is extremely high compared to traditional interfaces and is decreased by the presence of air, in contrast to measurements on larger, rougher interfaces. The data suggests that trapped interstitial gas at nanometer roughness serves primarily to reduce the solid-solid contact area, overwhelming its traditional role in providing alternate conduction paths.

\section{BACKGROUND}

Given the experimental difficulties of mechanically handling very small devices, measurements of the TCC of microstructures cannot be performed in the same way as is done on the macroscale. Large samples are often measured using a mechanical press that brings two cylindrical bars together with a specified force[7-9]. The bars are often contained within a vacuum chamber so that the interface can be tested under vacuum in order to isolate a value for the solid-solid conductance in the absence of the interstitial gas. Heat is applied to one cylinder and a series of thermocouples measure the temperature along the axial direction of the bars

For micromachined structures, one does not have direct mechanical control of the interface, but rather one must move it indirectly through the application of a voltage (assuming electrostatic actuation). A technique commonly used to calculate the thermal conductance of bolometers[10] and micromirrors[11] can be modified to obtain the TCC of actuated interfaces. Consider a square microstructure supported by two long thin beams, as shown in Fig. 1. The microstructure is designed such that the plate has a moderate resistance and the beams have negligible resistance. If a current flows through the device, then the dissipated power is $P_{\text {in }}=I^{2} R$. The resistance, $R$, depends on temperature via the equation, $R=R_{0}[1+\alpha \Delta T]$, where $R_{0}$ is initial resistance at ambient temperature, $\alpha$ is the temperature coefficient of resistance (TCR), and $\Delta \mathrm{T}$ is the difference in temperature between the device and its surroundings. If the microstructure is designed so that its supports are of uniform cross-section, then the heat dissipated out of the structure will be $P_{\text {out }}=G \Delta T$, where $G$ is the thermal conductance in $\mathrm{W} / \mathrm{K}$. In steady-state, $\mathrm{P}_{\text {in }}=\mathrm{P}_{\text {out }}$, and we can write the expression,

$$
\frac{1}{R}=\frac{1}{R_{0}}-\frac{\alpha}{G} I^{2}
$$

which relates the slope of $1 / \mathrm{R}$ vs. $\mathrm{I}^{2}$ to the thermal conductance of a microstructure. By designing a microstructure to have very low thermal conductance when suspended above the surface of a substrate, one can make the TCC the dominant heat transfer mechanism when the device is actuated. The measurement of $\mathrm{G}$ is then a probe of TCC. An image of the apparatus used in these measurements is shown in Fig. 2.

\section{EXPERIMENT AND DISCUSSION}

The test structures used to measure the TCC are fabricated in a standard surface micromachining process. First a $500 \mathrm{~nm}$ silicon nitride layer is deposited on top of a silicon substrate followed by $1500 \mathrm{~nm}$ of silicon dioxide, which is patterned and etched to form anchor points for a $750 \mathrm{~nm}$ thick doped polysilicon layer. The polysilicon is etched into square plates supported by two $24 \mu \mathrm{m}$ wide beams. The beams vary in length from $50 \mu \mathrm{m}$ to $150 \mu \mathrm{m}$, and the plates vary in size from $150 \mu \mathrm{m}$ to $200 \mu \mathrm{m}$ square. The support beams are coated with gold to insure that the vast majority of the device resistance occurs in 
the plate. The various geometric and material parameters for the structures are shown in Table I.

If a plate in air is not actuated, the thermal conductance, $\mathrm{G}$, is dominated by air conduction. For example, a $200 \mu \mathrm{m}$ square plate in air as shown in Fig. 1 has a measured conductance of $1.77 \times 10^{-4} \mathrm{~W} / \mathrm{K}$. Under vacuum, a structure is dominated by conduction through the support arms. Fig. 4 shows that the vacuum conductance for a $200 \mu \mathrm{m}$ plate with $75 \mu \mathrm{m}$ supports in vacuum is $1.77 \times 10^{-5} \mathrm{~W} / \mathrm{K}$. When a structure is snapped-down, the thermal conductance is dominated by the TCC, as seen by the greater than an order of magnitude change in both air and vacuum in Figs. 3 and 4. The magnitude of the TCCs in air and vacuum are about $6.0 \times 10^{4} \mathrm{~W} /\left(\mathrm{K}-\mathrm{m}^{2}\right)$ and $9.5 \times 10^{4} \mathrm{~W} /\left(\mathrm{K}-\mathrm{m}^{2}\right)$, respectively. Other samples can vary by as much as a factor of 2 from these values but the air/vacuum trend is always the same. All of these numbers are significantly greater than that seen with traditional interfaces, even compared to metal-metal structures $[6,8,9,12]$. The pressure of the contact can be extracted from the applied voltage at actuation, which corresponds to a force of $F=\varepsilon A V^{2} / d^{2}$, where $F$ is the force, $\varepsilon$ is the dielectric constant of silicon nitride, $\mathrm{A}$ is the contact (plate) area, $\mathrm{V}$ is the applied voltage, and $\mathrm{d}$ is the gap spacing defined by the thickness of the silicon nitride insulator. A typical value for an applied voltage of $65 \mathrm{~V}$ is about $1 \mathrm{MPa}$. The samples show a monotonic increase in TCC with pressure in both vacuum and air, as expected from bulk theory.

In addition to providing quantitative data for the TCC, Figs. 3 and 4 show the surprising result that the TCC is higher when the structures are actuated in a vacuum. This behavior is very different from bulk interfaces where the TCC decreases in vacuum. For any given test structure, it is found that the TCC is smallest when the device is snapped down in air, increases if the vacuum chamber is pumped out with the device snapped down, and increases still further if the structure is then released and reactuated. An example is shown in Table 2. Fig. 6 shows results for the TCC vs. increasing pressure where the pressure is applied electrostatically by increasing the voltage after snap-down. The TCC increases continuously with pressure as expected from theory.

Based on these observations, we propose that this behavior is due to trapped air in the small interstitial voids, whose primary contribution to the TCC is reduced solid-solid contact area rather than the provision of an efficient alternate conduction path. In-use stiction studies provide some independent support for this explanation. While very few of the test structures become stuck to the substrate during repeated actuation in air, this behavior occurs to the majority of devices actuated in vacuum. Since stiction is related to the Van der Waals interactions of contact points at the interface, the difference in stiction for an individual device suggests a difference in solid-solid contact area.

One probable origin of these behaviors is the relative surface roughness of the interfaces. As mentioned in the introduction, most previous TCC studies have been done on samples with surface roughness on the order of 0.1 to $10 \mu \mathrm{m}$. The roughness of the samples used in this study is on the order of a few nanometers. For example, the structure whose data is shown in Figures 3 and 4 has a roughness of $8 \mathrm{~nm}$, as measured by optical interferometry. It is interesting to speculate that the contact area and TCC at such small roughnesses may be dominated more by the energy and viscosity of the interfacial fluid than by the topography of the interface itself.

\section{CONCLUSIONS}

The TCC of micromechanical interfaces has been measured electronically, using the change in resistance with current as a probe of the underlying thermal conductivity. From these measurements it is found that the TCC of microactuated interfaces, which have a film roughness on the order of nanometers, is significantly higher than seen in rougher macroscopic interfaces. Surface treatments, such as selfassembling monolayers, had little effect on the TCC. In addition, the TCC of micromachined completely differently in the presence of an interstitial gas (air), namely the conductance increases as the interstitial gas is removed from the interface. Measurements suggest that the air reduces the solid-solid contact area, superseding its traditional role in providing an alternative conduction path.

\section{REFERENCES}

1. See for example, A. D. Kraus and A. Bar-Cohen, Thermal Analysis and Control of Electronic Equipment, McGraw-Hill, 1983, pp. 199-214.

2. W. P. King, T.W. Kenny, K.E.Goodson, et. al., "Atomic force microscope cantilevers for combined thermomechanical data writing and reading," Applied Physics Letters, 78, 1300, (2001).

3. R. A. Wood, "Monolithic silicon microbolometer arrays," Ch. 3 in Uncooled Infrared Imaging Systems and Arrays, Ed. P. W. Kruse, and D. D. Skatrud, Semiconductors and Semimetals, vol. 47, Academic Press, New York, 1997, pp. 43-121.

4. R.N. Supino and J. J. Talghader, "Electrostatic control of microstructure thermal conductivity," Applied Physics Letters, 78, 1778, (2001).

5. S. M. S. Wahid, and C. V. Madhusudana, "Gap conductance in contact heat transfer", International Journal of Heat and Mass Transfer, 43, 4383 (2000).

6. J. J. Salgon, F. Robbe-Valloire, J. Blouet, and J. Bransier, "A mechanical and geometrical approach to thermal contact resistance," International Journal of Heat and Mass Transfer, 40, 1121 (1997).

7. L. S. Fletcher, P. A. Smuda, and D. A. Gyorog, American Institute of Aeronautics and Astronautics Journal, 7, 1302 (1969).

8. K. E. Parmenter and E. Marschall, "Influence of surface preparation on thermal contact conductance of stainless steel and aluminum," Experimental Heat Transfer, 8, 195 (1995).

9. E. G. Wolff and D. A. Schneider, "Prediction of thermal contact resistance between polished surfaces," International Journal of Heat and Mass Transfer, 41, 3469 (1998).

10. P. Eriksson, J. Y. Andersson, and G. Stemme, "Thermal Characterization of Surface-Micromachined Silicon Nitride Membranes for Thermal Infrared Detectors," Journal of Microelectromechanical Systems, 6, 55, (1997).

11. R. N. Supino and J. J. Talghader, "Average optical power monitoring in micromirrors," IEEE Journal of Selected Topics in Quantum Electronics, 8, 1 (2002).

12. T. Aikawa and W. O. Winer, "Thermal contact conductance across $\mathrm{Si}_{3} \mathrm{~N}_{4}-\mathrm{Si}_{3} \mathrm{~N}_{4}$ contact," Wear, 177, 25 (1994). 


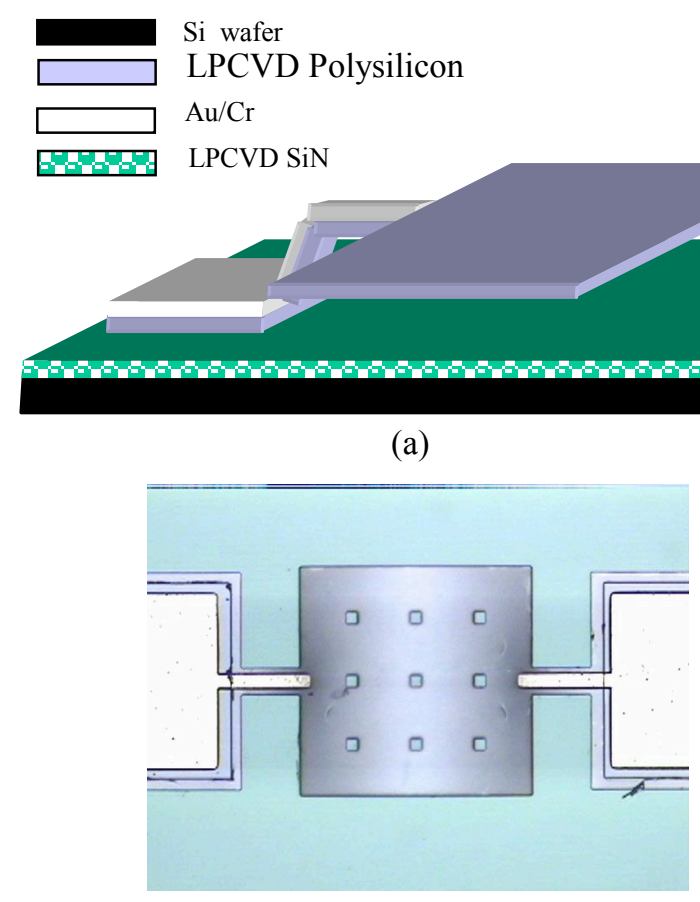

(b)

Fig. 1 - Diagram(a) and optical micrograph(b) of a test structure used in this study to measure thermal contact conductance.

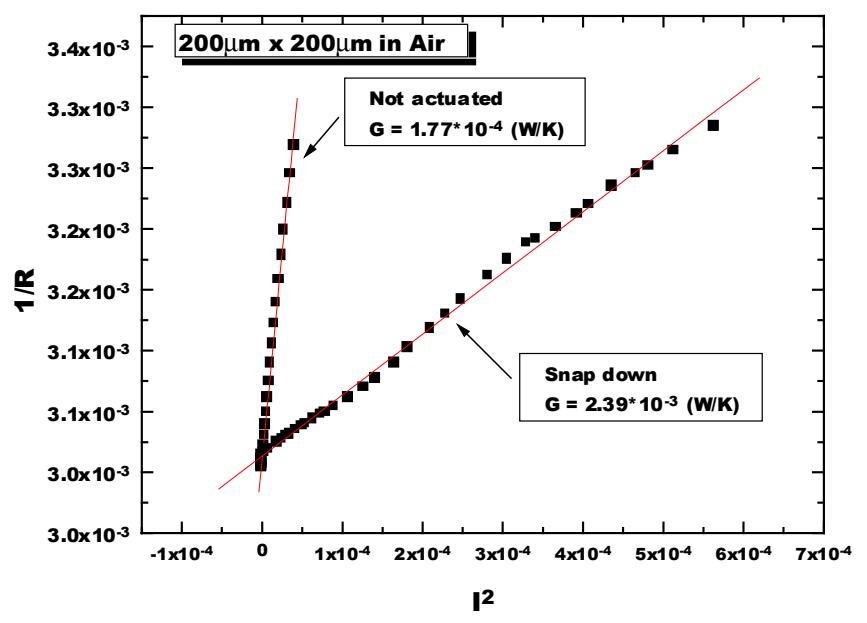

Fig. 3 - 1/R vs, $I^{2}$ for a test structure in air. The relatively flat curve is the measurement for the unactuated device, while the steeply sloped curve is for the snap-down condition. The slope of the lines is inversely proportional to the thermal conductance. Note that the TCC measured in this manner ( $\mathrm{G}$ under snap-down) is more than an order of magnitude greater than typical values seen with bulk metal interfaces. At least part of this difference is likely due to the extreme flatness $(<2 \mathrm{~nm})$ of the polysilicon and nitride surfaces.

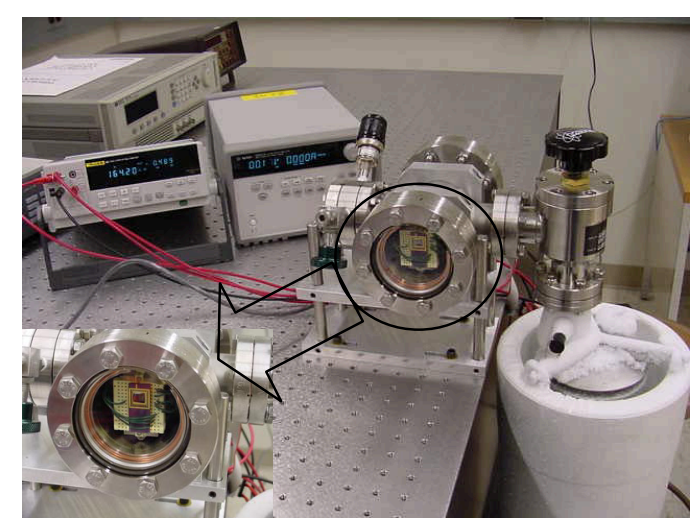

Fig. 2 - Image of vacuum system used to measure TCC. The optical window allows optical measurements to be made in addition to electrical ones.

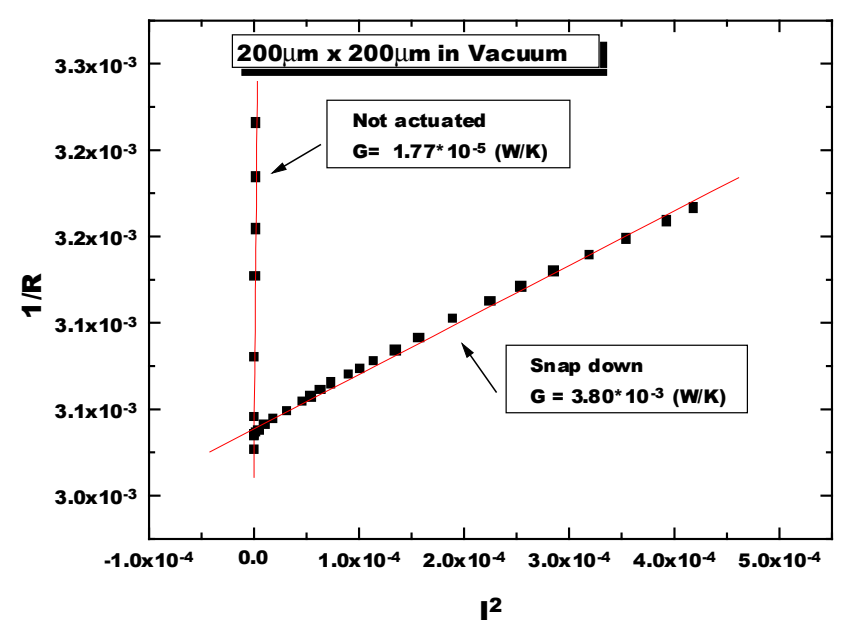

Fig. 4 - 1/R vs, $I^{2}$ for the test structure in Fig. 3 in vacuum. The relatively flat curve is the measurement for the unactuated device, while the steeply sloped curve is for the snap-down condition. Note that the TCC in vacuum ( $G$ under snap-down) is significantly higher than the TCC in air from Fig. 3. This behavior is different from that of bulk interfaces. It implies that the interstitial fluid in actuated MEMS devices reduces the solid-solid contact area of the polysilicon-nitride interface. The alternate conduction path provided by the air is not efficient enough to overcome the reduction in contact area. 


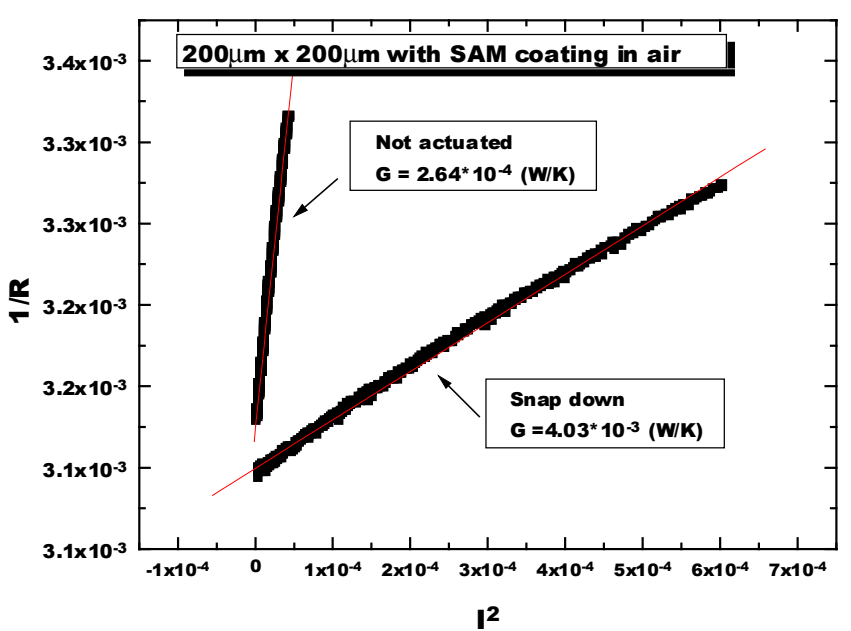

Fig. 5 - 1/R vs, $I^{2}$ for a SAM-coated test structure in air. The relatively flat curve is the measurement for the unactuated device, while the steeply sloped curve is for the snap-down condition. The snap-down voltage is $50 \mathrm{~V}$. The TCC measured for this device (and others) falls within the range of typical TCCs measured for devices without the SAM.

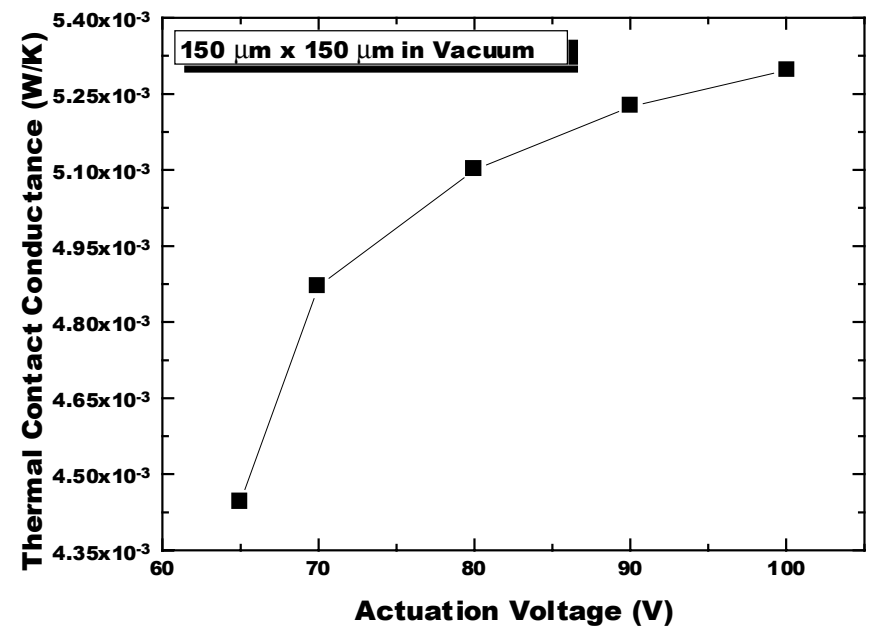

Fig. 6 - Thermal conductance vs. actuation voltage for a test structure in vacuum. All of the applied voltages are greater than the snap-down condition. The increased voltage across the insulator causes increased attractive force, so this curve is an indirect measurement of the TCC vs. pressure.

\begin{tabular}{|c|c|}
\hline Parameter & Value \\
\hline Size of Mirror & $\begin{array}{c}200 \mu \mathrm{m} \times 200 \mu \mathrm{m} \text { and } \\
150 \mu \mathrm{m} \times 150 \mu \mathrm{m}\end{array}$ \\
\hline Thickness of Mirror & $0.75 \mu \mathrm{m}$ \\
\hline Length of supporting arm & $75 \mu \mathrm{m}$ and $100 \mu \mathrm{m}$ \\
\hline Width of supporting arm & $24 \mu \mathrm{m}$ \\
\hline Air Gap Spacing & $1.5 \mu \mathrm{m}$ \\
\hline Thermal Conductivity of Polysilicon $(\mathrm{G})$ & $0.29 \mathrm{~W} /(\mathrm{cm}-\mathrm{K})$ \\
\hline Thermal Conductivity of dry air at $20^{\circ} \mathrm{C}$ & $0.0002564 \mathrm{~W} /(\mathrm{cm}-\mathrm{K})$ \\
\hline Density of Polysilicon & $2.3 \mathrm{~g} / \mathrm{cm}^{3}$ \\
\hline Specific heat of Polysilicon & $0.92 \mathrm{~J} /(\mathrm{g}-\mathrm{K})$ \\
\hline Temperature Coefficient of Resistance $(\mathrm{a})$ & $-0.12 \%\left(\mathrm{~K}^{-1}\right)$ \\
\hline Resistance (R), Basic Resistance $\left(\mathrm{R}_{0}\right)$ & at $\mathrm{T}_{0}$, and Current $(\mathrm{I})$ \\
\hline
\end{tabular}

Table 1 - Parameters and variable definitions used in this study.
Thermal Contact Conductance of $150 \mu \mathrm{m}$ x $150 \mu \mathrm{m}$ mirror (unit: $\mathrm{W} / \mathrm{K}$ )

\begin{tabular}{lc}
\hline In Air with the mirror snapped down & $2.69 \times 10^{-3}$ \\
\hline $\begin{array}{l}\text { In pumping out with the mirror } \\
\text { snapped down }\end{array}$ & $2.94 \times 10^{-3}$ \\
\hline In vacuum, released and re-actuated & $3.10 \times 10^{-3}$ \\
\hline
\end{tabular}

Table 2 - TCC of a $150 \mu \mathrm{m} \times 150 \mu \mathrm{m}$ test structure in air and in vacuum. The test structure is first snappeddown and measured in air. While still snapped down, the vacuum chamber is pumped out (to below 50mTorr) and allowed to stabilize. The TCC is then remeasured and found to be higher. The device is released and then reactuated, and the TCC is found to be yet higher. These measurements may be explained if some interstitial fluid (air) is trapped under the device by the actuation and then released when the actuation voltage is removed. The effect is even larger for larger area devices. 